\title{
Optimization of 2-D structures subjected to nonlinear deformations using the homogenization method
}

\author{
K. Yuge \\ Department of Mechanical Engineering, Seikei University, Kichijyoji-Kita 3, Musashino-Shi Tokyo 180-8633, Japan \\ N. Iwai \\ Department of Mechanical Engineering, University of Kentucky, Lexington, KY 40506-0108, USA
}

\section{N. Kikuchi}

Department of Mechanical Engineering and Applied Mechanics, The University of Michigan, Ann Arbor, MI 48109-2215, USA

\begin{abstract}
The generalized layout optimization method is applied to nonlinear problems. The algorithm was originally invented by Bendsøe and Kikuchi (1988), where an admissible design domain is assumed to be composed of periodic microstructures with tiny cavities; the sizes and the rotational angle of the cavities are defined as design variables which are optimized to minimize the applied work. The macroscoic material tensor of the porous material is calculated by the homogenization method for the sensitivity analysis. In order to apply it to nonlinear problems, we present a 2-D database of the material tensor calculated by the elasto-plastic homogenization method and an interpolation technique of the database for the practical computation. Several numerical examples of 2-D structures and a thin shell are shown to demonstrate the effectiveness of our algorithms. The algorithm is also extended to the finite deformation problems, and a practical optimized design is exhibited.
\end{abstract}

\section{Introduction}

The generalized layout optimization method, proposed by Bendsøe and Kikuchi (1988), can determine the optimal design for the topology and shape problems. In this method, periodic microstructures with rectangular cavities are assumed in the admissible domain; the sizes and rotational angle of the cavities are specified as the design variables to minimize the applied work. For the sensitivity analysis, the macroscopic material tensor of the porous material is computed by the homogenization method presented by Lions (1981), and Guedes and Kikuchi (1990).

Several optimized designs of the elastic structures computed by the method were exhibited by Suzuki and Kikuchi (1991). The algorithm was applied to the elastic shell by Suzuki and Kikuchi (1992) and extended into dynamic problems by Diaz and Kikuchi (1992); Ma et al. (1993, 1995). Yuge and Kikuchi (1995) developed the optimization for a frame structure subjected to a nonlinear deformation, where the elasto-plastic homogenization method formulated by Terada et al. (1995); Terada et al. (1996) was used.
In this paper, the generalized layout optimization method is applied to 2-D structures and a thin shell subjected to plastic deformation. Firstly, the main idea of the optimization is briefly discussed. In order to apply the method to nonlinear problems, a database of the macroscopic material tensor is presented using the elasto-plastic homogenization method and its interpolation technique is proposed for the sensitivity analysis. It is shown by numerical examples for 2-D problems that the plastic collapse load of the optimized structure becomes much higher than that of the initial design. It is also exhibited that the optimized designs for nonlinear and linear problems are different from each other. Secondly, our method is applied to a thin shell and a numerical example is demonstrated. The numerical example displays that the optimal material distribution is determined to sustain plastic deformation in the whole admissible design domain. Finally, the method is applied to the finite deformation infinitesimal strain problems, and a practical design of the reinforcement member of a structure is presented to demonstrate the effectiveness of our algorithm.

\section{Algorithm}

In this section, the main idea of the generalized optimization method is briefly explained. Then application to twodimensional nonlinear problems is presented.

\subsection{Main idea of the optimization}

Suppose an admissible design domain is composed of periodic microstructures with cavities, as shown in Figs. 1a and b. Boundary conditions and applied loads are given to the macroscopic structure. After the sensitivity analysis, the cavity sizes and the angles of the microstructures are determined to increase the stiffness of the macroscopic structure. That is to say, the cavity sizes are reduced in areas with high strain energy density and increased in areas with low strain energy density; the angles are rotated to the same angles of the principal stresses, as shown in Figs. 1c and d, respectively. After 
the iteration for the optimization, we can obtain the microscopic and the macroscpic layouts simultaneously, as depicted in Figs. 1b-d. For the sensitivity analysis, the macroscopic material tensor for the porous material is computed by the homogenization method. For the practical calculation of nonlinear problems, we construct the database of the material tensor and present the interpolation technique.

In this paper, a size of the cavity (1-a) and a rotational angle of the microstructures $\theta$ are defined as design variables assuming microstructures with square cavities, square-type microstructures, as shown in Fig. 2a. Not requiring the third parameter (1-b) in rectangular-type microstructures presented by Bendsøe and Kikuchi (1988), as shown in Fig. 2b, carries a great advantage for making the database of the homogenized material tensor. If linear elastic problems are assumed, triangular-type microstructures, as shown in Fig. 2c, have more of an advantage since they have isotropy and require the only parameter (1-a). But it was shown by Yuge and Kikuchi (1996) that the isotropy disappeared when plastic deformation was applied. In addition, Yuge et al. (1997) exhibited that the ultimate strength of the optimized design with triangular-type microstructures was slightly less than that with square-type microstructures. Therefore, we employ square-type microstructures and design variables are (1-a), and $\theta$.

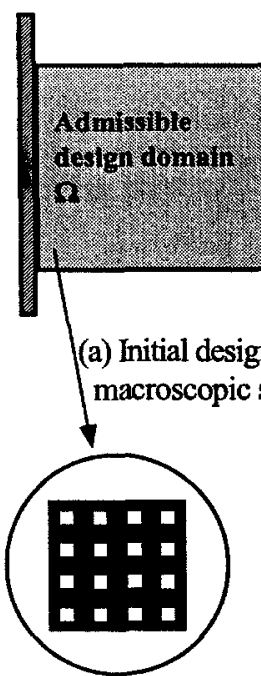

(b) Initial distribution of microstructures

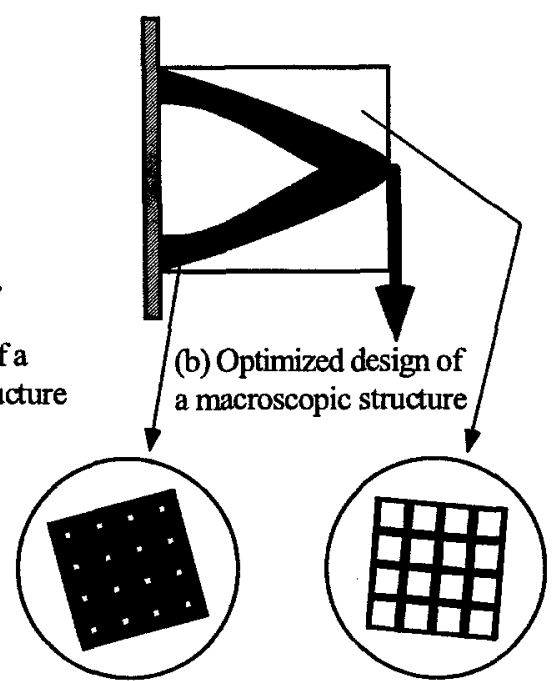

(c) Microstructures for large strain aera (d) Microstructures for small strain area
Fig. 1. Optimization by the homogenization method. (a) Initial design of a macroscopic structure; (b) optimized design of a macroscopic structure; (b) initial distribution of microstructures; (c) microstructures in areas with high strain energy density; (d) microstructures in areas with low strain density

The optimization problem with constraints is defined as

minimize $F$ subjected to $h \leq 0, \underline{a} \leq a_{i} \leq \bar{a}$.

External work done by the applied load is defined as an objective function $F$, expressed by

$F=\iint_{\Omega}^{u} \mathbf{f} \cdot \mathrm{d} \mathbf{u} \mathrm{d} \Omega=$ $\mathbf{y}=\mathbf{x} / \varepsilon$

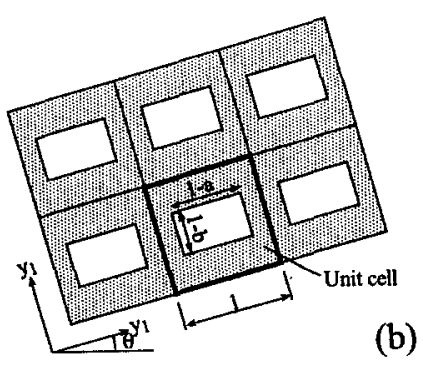

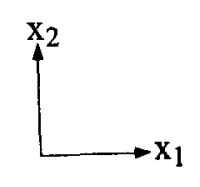

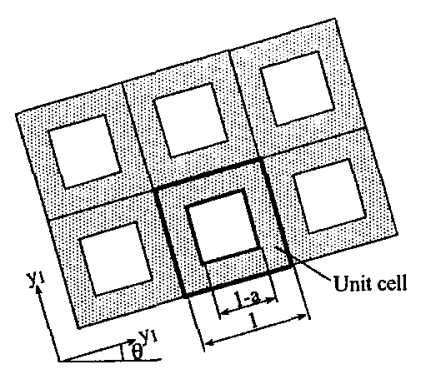

(a)

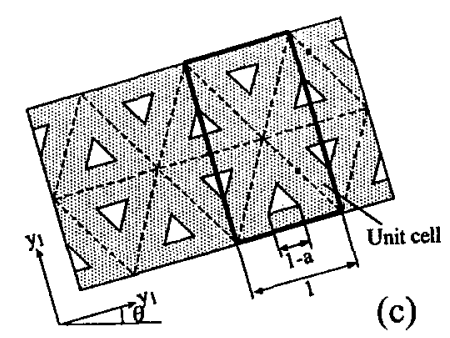

Fig. 2. Design variables in microstructures with periodic cavities. (a) Square type, (b) rectangular-type employed by Bendsøe and Kikuchi, (c) regular triangular type

$$
\sum_{j=1}^{N \text { step }}\left\{\int_{\Omega} \frac{1}{2}\left[\mathbf{f}^{(j-1)}+\mathbf{f}^{(j)}\right] \cdot \Delta \mathbf{u}^{(j)} \mathrm{d} \Omega\right\} .
$$

In (2), $\mathbf{f}$ denotes the external force vector, $\mathbf{u}$ represents the displacement vector, $\Delta \mathbf{u}$ indicates the incremental displacement by the applied force increment, $j$ expresses the $j$-th load-incremental step, and $N$ step means the total number of the load-incremental steps. Bendsøe et al. (1993) interpreted that the objective function $F$ is minimized when a prescribed force is given to the structure, and it is maximized when a prescribed displacement is applied. The constraint of the total amount of material, $h$, is expressed by

$h=\int_{\Omega}\left\{1-(1-a)^{2}\right\} \mathrm{d} \Omega-\Omega_{0}=$

$$
\sum_{i=1}^{N E} \int_{\Omega_{e}}\left\{2 a_{i}-a_{i}^{2}\right\} \mathrm{d} \Omega-\Omega_{0} \leq 0 .
$$

In (3), $a_{i}$ represents the parameter of the cavity size, which is determined as a constant value in an element; subscript $i$ means element number; $N E$ denotes the total number of the global elements; $\Omega_{0}$ expresses the total volume of the material which can be used in the admissible design domain $\Omega$. The minimum and the maximum value of $a_{i}$ are expressed by $\underline{a}$ and $\bar{a}$ (i.e. $\underline{a}=0, \bar{a}=1.0$ ).

The Lagrange function for the constrained optimization problem is defined as

$$
L=F+\lambda h+\sum_{i=1}^{N E}\left\{\alpha_{i}\left(\underline{a}-a_{i}\right)+\beta_{i}\left(\bar{a}-a_{i}\right)\right\}
$$


where $\lambda, \alpha$, and $\beta$ are Lagrange multipliers. The KuhnTucker conditions for the optimized solution are expressed by

$\frac{\partial F}{\partial a_{i}}+\lambda \frac{\partial h}{\partial a_{i}}=\alpha_{i}-\beta_{i} \quad(i=1,2, \ldots, N E)$,

$\lambda h=0$,

$\alpha_{i}\left(\underline{a}-a_{i}\right)=0 \quad(i=1,2, \ldots, N E)$,

$\beta_{i}\left(\bar{a}-a_{i}\right)=0 \quad(i=1,2, \ldots, N E)$

$\lambda \geq 0, \alpha_{i} \geq 0, \beta_{i} \geq 0 \quad(i=1,2, \ldots, N E)$.

According to Yuge and Kikuchi (1995), the sensitivity of $F$ with respect to $a_{i}$ in (5) is expressed as,

$\frac{\partial F}{\partial a_{i}}=-\frac{\partial \int_{\Omega} \int_{0}^{\varepsilon} \sigma: \mathrm{d} \varepsilon \mathrm{d} \Omega}{\partial a_{i}}=-\frac{\partial\left(\int_{\Omega} U_{\mathrm{int}} \mathrm{d} \Omega\right)}{\partial a_{i}}$.

In the above equation, $U_{\text {int }}$ denotes the internal work in the loading process. If we assume that the strain is small and increases monotonously, $U_{\text {int }}$ can be calculated by

$U_{\text {int }}=\sum_{j=1}^{N \text { step }}\left[\boldsymbol{\sigma}^{(j)}+1 / 2 \mathbf{C}^{H(j)}: \Delta \varepsilon\right]: \Delta \varepsilon$

where

$\sigma^{(j)}=\sum_{k=1}^{j}\left[\mathrm{C}^{H(k)}: \Delta \varepsilon\right]$

$\Delta \varepsilon=\frac{\varepsilon}{N \text { step }}$

In (13), $\varepsilon$ denotes total strain in the vicinity of the reference points at the end of the load-incremental analysis. The mathematically averaged nonlinear material tensor of the porous material $\mathrm{C}^{H}$ is computed by the homogenization method, which is summarized later. Subscripts $j$ and $k$ refer to incremental step numbers. Partial differential of $U_{\text {int }}$ with respect to $a_{i}$ is calculated by the finite difference method. The derivative of $h$ can be obtained by

$$
\begin{aligned}
& \frac{\partial h}{\partial a_{i}}=\frac{\partial}{\partial a_{i}} \sum_{i=1}^{N E} \int_{\Omega_{e}}\left(2 a_{i}-a_{i}^{2}\right) \mathrm{d} \Omega-\Omega_{0}= \\
& \int_{\Omega_{e}}\left(2-2 a_{i}\right) \mathrm{d} \Omega .
\end{aligned}
$$

The optimality criteria approach is adopted to satisfy the Kuhn-Tucker conditions. The numerical formulation is explained precisely by Bendsøe and Kikuchi (1988); Suzuki and Kikuchi (1991); Ma et al. (1993).

\subsection{Outline of the elasto-plastic FEM based on the homog- enization method}

The elasto-plastic FEM based on the homogenization method is conducted to obtain the averaged material tensor of the porous material. According to Yuge and Kikuchi (1995); Terada et al. (1995), Terada et al. (1996), the incremental formulation is summarized, where linearity is instantaneously assumed.

We suppose a macroscopic structure consists of periodic microstructures, as shown in Figs. $3 \mathrm{a}$ and $\mathrm{b}$. The microstructures are composed of unit cells, which are the smallest structures as far as they have periodic geometry, as depicted in Fig. 3c. The different scale coordinates are defined, $\mathbf{x}$ for the macroscopic structure, $\mathbf{y}$ for the microscopic structures. The relationship between $\mathbf{x}$ and $\mathbf{y}$ are specified by $\mathbf{y}=\mathbf{x} / \varepsilon$, where $\varepsilon$ is a very small number. This indicates that the microstructures (or the unit cells) are very small.

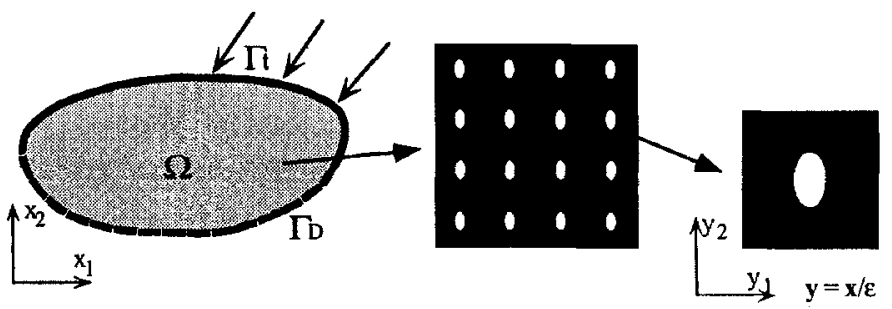
(a) Macroscopic structure
(b) Microstructures
(c) Unit cell

Fig. 3. Composite material with periodical microstructures. (a) Macroscopic structure, (b) micrcostructures, and (c) unit cell

The incremental displacement at an arbitrary point in the microstructures is supposed to be expressed as

$\Delta \mathbf{u}^{\varepsilon}(\mathbf{x}, \mathbf{y})=\Delta \mathbf{u}^{0}(\mathbf{x})+\varepsilon \Delta \mathbf{u}^{1}(\mathbf{x}, \mathbf{y})$.

Where $\Delta \mathbf{u}^{0}$ and $\Delta \mathbf{u}^{1}(\mathbf{x}, \mathbf{y})$ are the macroscopic (averaged) and the microscopic (local) displacement increment, respectively; $\Delta \mathbf{u}^{\mathbf{1}}(\mathbf{x}, \mathbf{y})$ is decomposed into

$\Delta \mathbf{u}^{1}(\mathbf{x}, \mathbf{y})=-\chi_{k}^{p q}(\mathbf{x}, \mathbf{y}) \frac{\partial \Delta u(\mathbf{x})_{p}^{0}}{\partial x_{q}}$

here $\boldsymbol{\chi}$ is the characteristic displacement in the microstructures.

The macroscopic weak form governing equation is given by

$\int_{\Omega} C_{i j k \ell}^{H}(\mathbf{x}) \frac{\partial \delta \Delta u_{i}^{0}}{\partial x_{j}} \frac{\partial \Delta u_{k}^{0}}{\partial x_{\ell}} \mathrm{d} \Omega=\int_{\Gamma} \delta \Delta u_{i}^{0} \Delta \sigma_{i j}^{T} n_{j} \mathrm{~d} \Gamma$.

In (17), the homogenized material tensor $\mathbf{C}^{H}(\mathbf{x})$ is defined by

$C_{i j k \ell}^{H}(\mathbf{x})=$

$\frac{1}{|\mathbf{y}|} \int_{\mathbf{y}}\left[\mathbf{C}_{i j k \ell}^{E P}(\mathbf{x}, \mathbf{y})-\mathbf{C}_{i j p q}^{E P}(\mathbf{x}, \mathbf{y}) \frac{\partial \chi_{p}^{k \ell}}{\partial y_{q}}\right] \mathrm{d} \mathbf{y}$ 
In (18), $\mathbf{C}^{E P}(\mathbf{x}, \mathbf{y})$ is the elasto-plastic material tensor of the solid material in the microstructures, which is defined by

$C_{i j k \ell}^{E P}=C_{i j k \ell}^{E}-y \frac{C_{i j a b}^{E} \sigma_{a b}^{\prime} C_{q r k \ell}^{E} \sigma_{q r}^{\prime}}{H^{\prime}+\sigma_{m n}^{\prime} C_{m n o p}^{E} \sigma_{o p}^{\prime}}$,

where

$\boldsymbol{\sigma}^{\prime}=\boldsymbol{\sigma}^{\varepsilon}(\mathbf{x}, \mathbf{y})-\frac{1}{3}\left[\operatorname{tr} \boldsymbol{\sigma}^{\varepsilon}(\mathbf{x}, \mathbf{y})\right] \mathbf{I}$,

$H^{\prime}=\frac{E E_{t}}{E-E_{t}}$.

In (19)-(21), $\mathbf{C}^{E}$ is the elastic material tensor; $\boldsymbol{\sigma}^{\boldsymbol{\varepsilon}}(\mathbf{x}, \mathbf{y})$ denotes the current stress in the microstructures; $E$ and $E_{t}$ are the modulus of elasticity and the tangent modulus, respectively. If the material is yielded, $y=1$, otherwise $y=0$. In (18), $\chi$ is obtained as the solution of the next weak form equation for the unit cell in the microstructures,

$\int_{\mathbf{y}} \frac{\partial \delta \Delta u_{i}^{1}}{\partial y_{j}}\left[C_{i j k \ell}^{E P}(\mathbf{x}, \mathbf{y})-C_{i j p q}^{E P}(\mathbf{x}, \mathbf{y}) \frac{\partial \chi_{p}^{k \ell}}{\partial y_{q}}\right] \mathrm{d} \mathbf{y}=0$

Calculation of $\Delta \sigma^{\varepsilon}(\mathbf{x}, \mathbf{y})$ is given by

$\Delta \sigma_{i j}^{\epsilon}(\mathbf{x}, \mathbf{y})=\left[C_{i j k \ell}^{E P}(\mathbf{x}, \mathbf{y})-C_{i j p q}^{E P}(\mathbf{x}, \mathbf{y}) \frac{\partial \chi_{p}^{k \ell}}{\partial y_{q}}\right] \frac{\Delta u_{k}^{0}}{x_{\ell}}$

According to the load-incremental method, the current stress and the displacement are calculated as

$\boldsymbol{\sigma}^{\varepsilon(j)}(\mathbf{x}, \mathbf{y})=\boldsymbol{\sigma}^{\varepsilon(j-1)}(\mathbf{x}, \mathbf{y})+\Delta \boldsymbol{\sigma}^{\varepsilon}(\mathbf{x}, \mathbf{y})$

$\mathbf{u}^{0(j)}(\mathbf{x}, \mathbf{y})=\mathbf{u}^{0(j-1)}(\mathbf{x}, \mathbf{y})+\Delta \mathbf{u}^{0}(\mathbf{x}, \mathbf{y})$

The numerical procedure of the above formulation is illustrated as follows:

1. Discretize the unit cells, in addition to the macroscopic structure,

2. Solve (22) for $\chi^{k \ell}$ of each degree of freedom in $\mathbf{y}$ (unit cell) with periodic boundary conditions,

3. Substitute $\chi^{k \ell}$ into (18), yielding the homogenized material tensor $\mathbf{C}^{H}$,

4. Solve the macroscopic equation (17) for $\Delta \mathbf{u}^{0}$ using $\mathbf{C}^{H}$,

5. Calculate $\boldsymbol{\sigma}^{\varepsilon(j)}(\mathbf{x}, \mathbf{y})$ using (23)-(24) and check yielding,

6. Repeat Steps 2 to 5 during load incremental steps.
2.3 Database of the homogenized material tensor and the interpolation technique

For effective computation of nonlinear problems, the homogenized material tensor $\mathrm{C}^{H}$ is determined by the interpolation of the database, where monotonous loading and small strain problems are assumed, since more than 99 percent of the computational time is consumed by the calculation of $\mathrm{C}^{H}$ if the homogenization method is directly used.

A thin plate subjected to biaxial loads is assumed to be a macroscopic structure, as shown in Fig. 4a. We suppose the macroscopic structure consists of square-type microstructures, as shown in Fig. 4b. The database for 2-D nonlinear problems has three parameters: cavity size $(1-a)$, combination of the macroscopic principal stresses $\left(\sigma_{1}, \sigma_{2}\right)$ and rotational angle to the one of the principal stress $\phi$, as defined in Fig. 4c. Discretizations of the unit cells with several cavity sizes are shown in Fig. 5 . The solid portion of the microstructures is assumed to be mild steel, that is, $E=205.8 \mathrm{MPa}$, $\sigma_{y}=294 \mathrm{MPa}, \nu=0.3$. The tangent modulus $E_{t}$ has a proportional limit at 80 percent of the yield stress, as shown in Fig. 6. If the microscopic equivalent stress exceeds the proportional limit, a plastic constitutive equation is adopted, $y=1$ in (19). Data points on the $\sigma_{1}-\sigma_{2}$ plane at specified " $a$ " and $\phi$ are shown schematically in Fig. 7a.

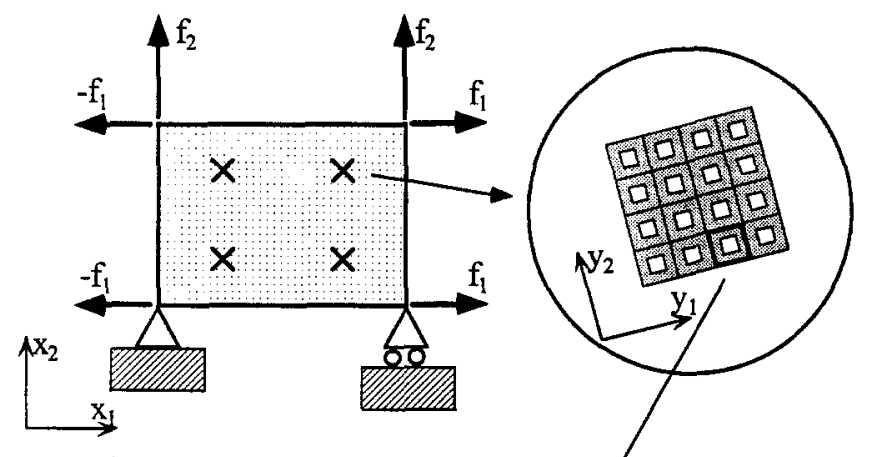

(a) Macroscopic structure (b) Microstructures

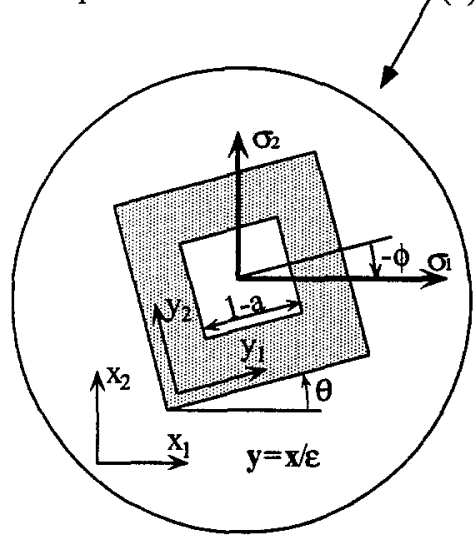

(c) Unit cell

Fig. 4. Thin plate subjected to biaxial loads. (a) Macroscopic structure, (b) microstructures, and (c) unit cell

The procedure of making the database is summarized as follows.

1. Conduct the elasto-plastic FEM based on the homoge- 


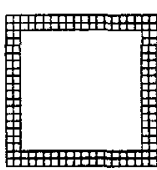

(a) $\mathrm{a}=0.2$

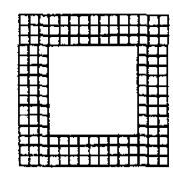

(b) $\mathrm{a}=0.4$

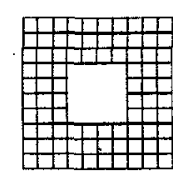

(c) $\mathrm{a}=0.6$

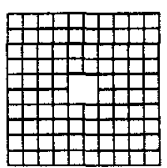

(d) $\mathrm{a}=0.8$

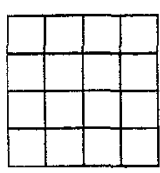

(e) $\mathrm{a}=1.0$
Fig. 5. Discretization of the unit cells. (a) $a=0.2$, (b) $a=0.4$, (c) $a=0.6$, (d) $a=0.8$, and (e) $a=1.0$

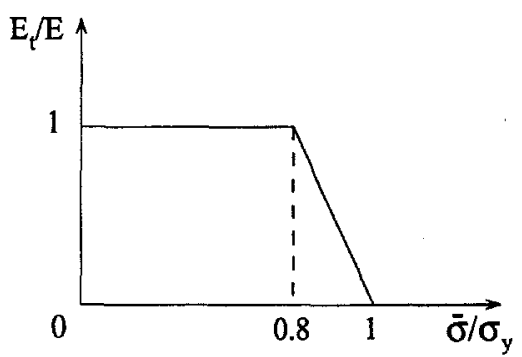

Fig. 6. Tangent modulus of solid material

nization method from $O$ to $H$ by way of $H^{\prime}$, shown in Fig. 7a. The innermost ellipse expresses the proportional limit, the outermost one stands for the place where the homogenized tangent modulus becomes zero.

2. Store $\mathbf{C}^{H}$ at the sampling points on the segment $H^{\prime}-H$. Figure $7 \mathrm{~b}$ exhibits examples of plastic areas of a unit cell at those sampling points.

3. Change the ratio of $\sigma_{1}$ to $\sigma_{2}\left(\sigma_{1}>\sigma_{2}\right)$ then repeat Steps 1 to 2 . We determine the ratios as $\sigma_{1}: \sigma_{2}=\cos \alpha: \sin \alpha$, where

$$
\alpha=-3 \pi / 4,-5 \pi / 8, \ldots, \pi / 4 \text {. }
$$

4. Change $\phi$, then repeat Steps 1 to 3 , specifying $\phi$ as $\phi=$ $-\pi / 4,-\pi / 8,0, \pi / 8$.

5. Change " $a$ ", then repeat Steps 1 to 4 , defining " $a$ " as $a=0.2,0.4,0.6,0.8,1.0$.

Figures 8a-d depict some examples of the yield surfaces of the obtained database, where the yield surfaces correspond with the outermost ellipse in Fig. 7a. It can be seen that the yield surfaces depend upon " $\phi$ " in addition to " $a$ ". Other examples calculated with the triangular-type microstructures are depicted for reference, as shown in Figs. 8e-f. It is observed that the yield surfaces are different from those of square-type microstructures, while the same parameters are used. It is also shown that both the square-type and the triangular-type microstructures have anisotropy for plastic problems. Figure 9 exhibits the histories of the yielded areas in the unit cells during the plastic deformation. You can easily see that the histories depend on the three parameters. Figure 10 shows a set of $(1,1,1,1)$ components of the obtained $\mathrm{C}^{H}$ with various combinations of the principal stresses at specified " $\phi$ " and " $a$ ". It should be noted that if linear elastic problems are assumed, only the parameter " $a$ " is required.

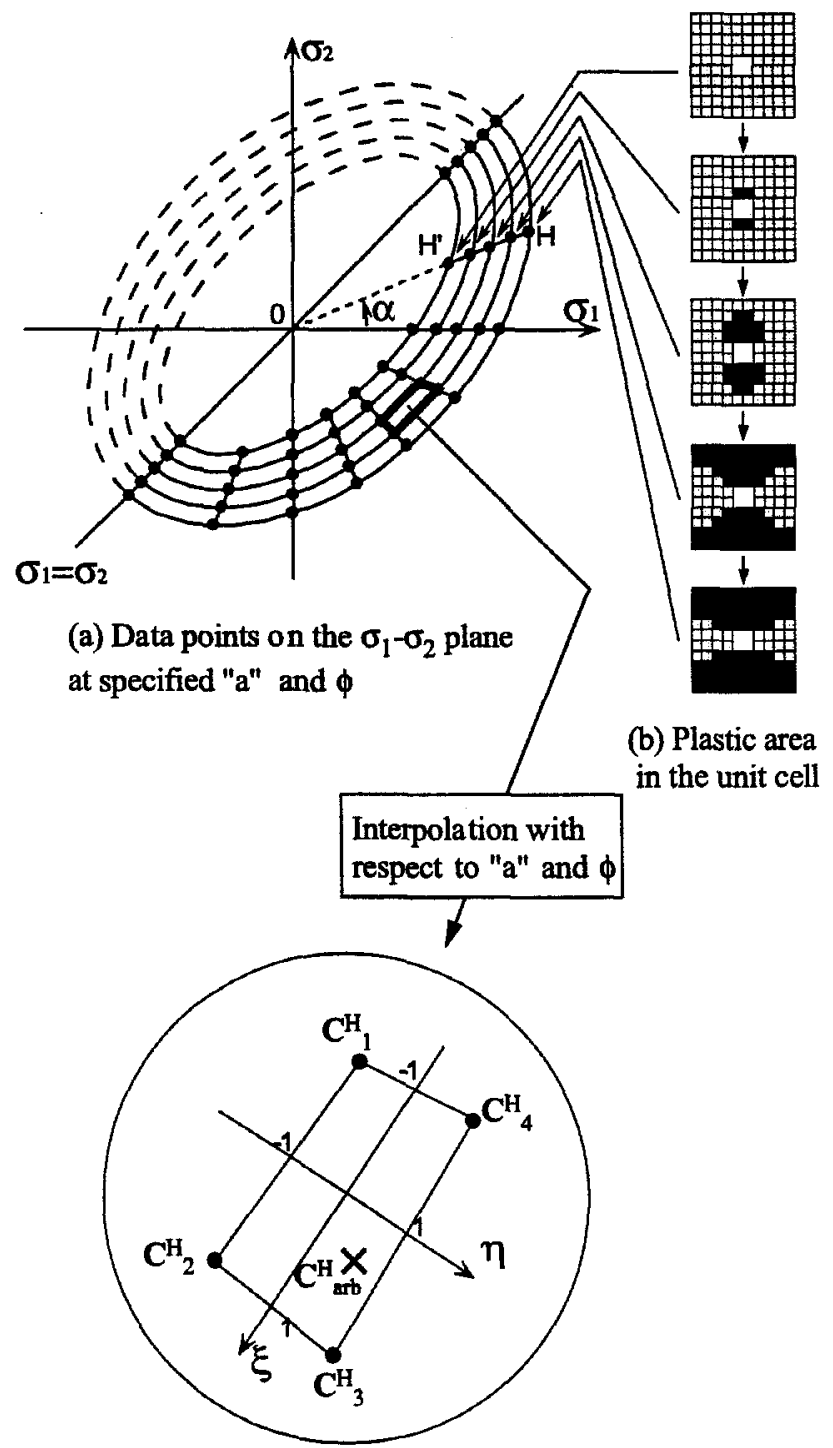

(c) Data points on the $\sigma_{1}-\sigma_{2}$ plane at a rbitrarily "a" and $\phi$

Fig. 7. Data points on a $\sigma_{1}-\sigma_{2}$ plane. (a) Data points on the $\sigma_{1}-\sigma_{2}$ plane at specified " $a$ " and $\phi$, (b) plastic areas in the unit cell, and (c) data points on the $\sigma_{1}-\sigma_{2}$ plane at arbitrary " $a$ " and $\phi$

\subsection{Interpolation of the database}

An interpolation technique of $\mathrm{C}^{H}$ for 2-D nonlinear problems is presented in this section. The interpolation has to be conducted at any iterative and incremental steps within the whole domain, because not only the design variables in the microstructures are updated within the whole macroscopic domain at every iterative step of the optimization, but constitutive equations are altered during the plastic deformation.

Linear interpolation with respect to " $a$ ", $\phi$ and $\left(\sigma_{1}, \sigma_{2}\right)$ is presented. This procedure is summarized as follows.

1. The interpolation with respect to " $a$ " is executed.

2. The coordinates transformation for the macroscopic stress is conducted by $\theta$, a rotational angle of microstructure 


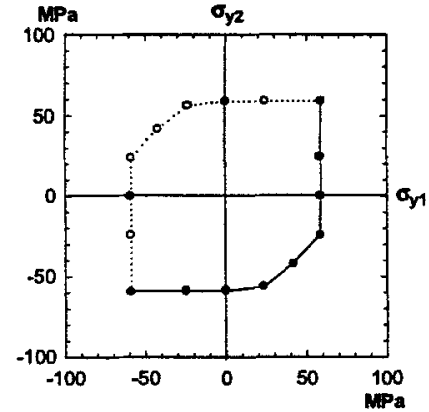

(a) $\mathrm{a}=0.2, \phi=0 \pi$

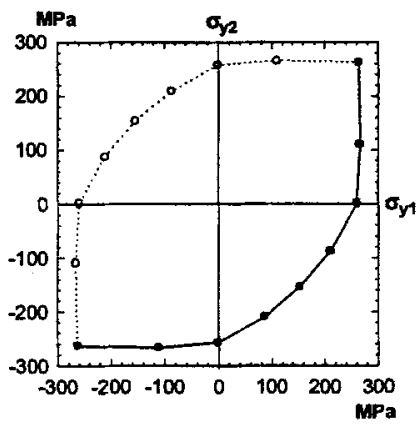

(c) $\mathrm{a}=0.8, \phi=0 \pi$

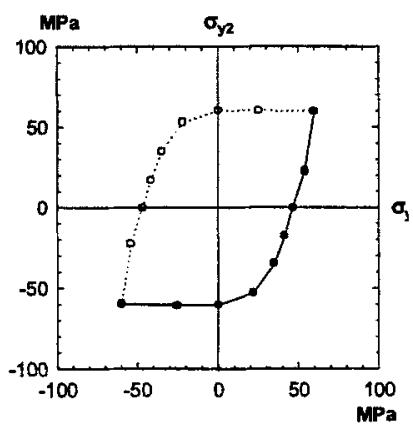

(e) $\mathrm{a}=0.2, \phi=0 \pi$, triangular-type

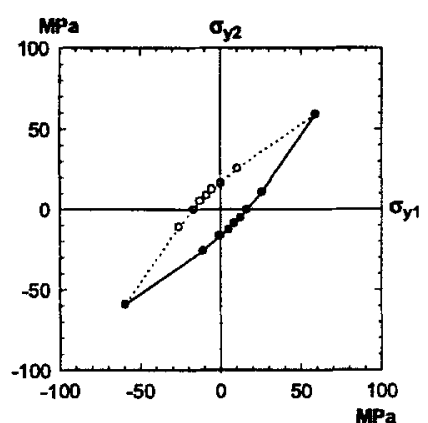

(b) $\mathrm{a}=0.2, \phi=-\pi / 4$

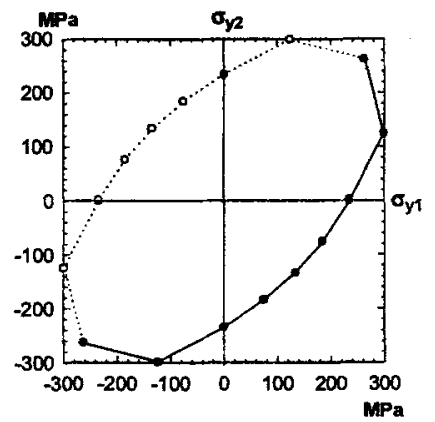

(d) $\mathrm{a}=0.8, \phi=-\pi / 4$

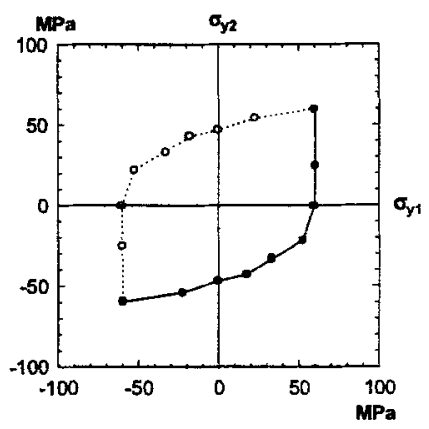

(f) $\mathrm{a}=0.2, \phi=-\pi / 6$, triangular-type
Fig. 8. Yield surfaces of the database, corresponding with the outer most data points. (a) $a=0.2, \phi=0 \pi$; (b) $a=0.2, \phi=$ $-\pi / 4$; (c) $a=0.8, \phi=0 \pi$; (d) $a=0.8, \phi=-\pi / 4$; (e) $a=0.2$, $\phi=0 \pi$, triangular-type; and (f) $a=0.2, \phi=-\pi / 6$, triangulartype

with respect to the macroscopic coordinate (Fig. 4c). Where

$\sigma_{\theta}=\mathbf{T}(\theta) \boldsymbol{\sigma}$,

$$
\begin{aligned}
& \mathbf{T}(\theta)=\left[\begin{array}{ccc}
\cos ^{2} \theta & \sin ^{2} \theta & 2 \cos \theta \sin \theta \\
\sin ^{2} \theta & \cos ^{2} \theta & -2 \cos \theta \sin \theta \\
-\cos \theta \sin \theta & \cos \theta \sin \theta & \cos ^{2} \theta-\sin ^{2} \theta
\end{array}\right] \\
& \boldsymbol{\sigma}=\left\{\sigma_{11}, \sigma_{22}, \sigma_{12}\right\}^{T} .
\end{aligned}
$$

3. The parameters $\phi$ and $\left(\sigma_{1}, \sigma_{2}\right)$ are calculated as follows:

$$
\phi=\frac{1}{2} \tan ^{-1}\left(\frac{-2 \sigma_{\theta, 12}}{\sigma_{\theta, 11}-\sigma_{\theta, 22}}\right),
$$

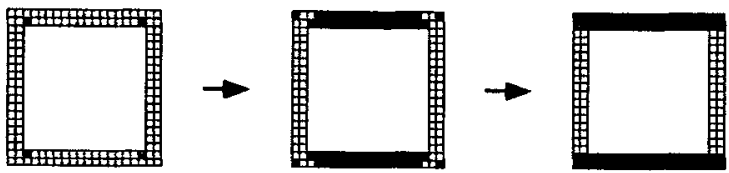

(a) $\mathrm{a}=0.2, \phi=0 \pi, \sigma_{1}: \sigma_{2}=1: 0$

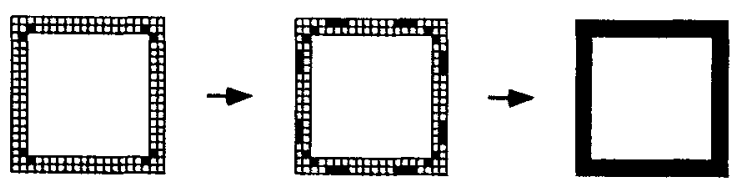

(b) $\mathrm{a}=0.2, \phi=0 \pi, \sigma_{1}: \sigma_{2}=1: 1$

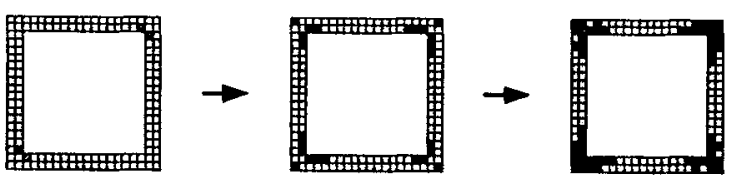

(c) $\mathrm{a}=0.2, \phi=-\pi / 4, \sigma_{1}: \sigma_{2}=1: 0$

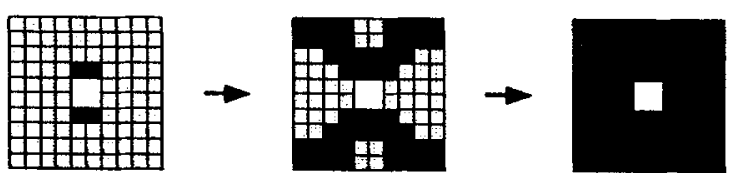

(d) $\mathrm{a}=0.8, \phi=0 \pi, \sigma_{1}: \sigma_{2}=1: 0$

Fig. 9. Histories of the plastic areas in the unit cells. (a) $a=0.2$, $\phi=0 \pi, \sigma_{1}: \sigma_{2}=1: 0$; (b) $a=0.2, \phi=0 \pi, \sigma_{1}: \sigma_{2}=1: 1$; (c) $a=0.2$, $\phi=-\pi / 4, \sigma_{1}: \sigma_{2}=1: 0 ;$ (d) $a=0.8, \phi=0 \pi, \sigma_{1}: \sigma_{2}=1: 0$

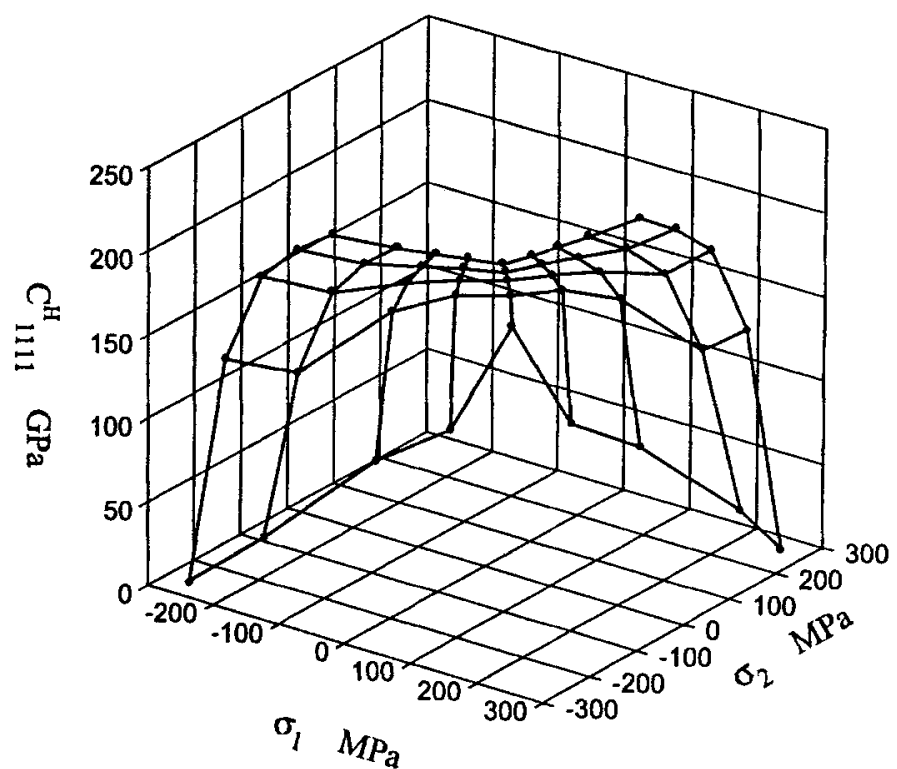

Fig. 10. Part of the data base on a $\sigma_{1}-\sigma_{2}$ plane $(a=0.8, \phi=0)$

$$
\sigma_{1}, \sigma_{2}=\frac{\sigma_{\theta, 11}+\sigma_{\theta, 22}}{2} \pm \sqrt{\left(\frac{\sigma_{\theta, 11}+\sigma_{\theta, 22}}{2}\right)^{2}+\sigma_{\theta, 12}^{2}}
$$

4. The database is interpolated with respect to $\phi$. Noting that the microstructures have symmetry of $\pi / 2$ rotation, 
$\phi$ is transformed into $\phi^{\prime}=\phi-n \pi / 2$ where $-\pi / 4 \leq \phi^{\prime} \leq$ $\pi / 4$, if necessary.

5. The material tensor for the arbitrary combination of the principal stresses, $\mathrm{C}_{\mathrm{arb}}^{\mathrm{H}}$, is given by

$\mathrm{C}_{\mathrm{arb}}^{H}=\sum_{j=1}^{4} N_{j}(\xi, \eta) \mathbf{C}_{j}^{H}$

as shown in Fig. 7c. Where $N_{j}(\xi, \eta)$ are shape functions. Nondimensional parameters $\xi$ and $\eta$ are determined by the Newton-Raphson method. Note that $\mathbf{C}_{j}^{H}$ on the $\sigma_{1}-$ $\sigma_{2}$ plane, as shown in Fig. $7 \mathrm{c}$, has been interpolated with respect to " $a$ " and $\phi$.

6. If $\phi^{\prime}$ is substituted for $\phi$ by $\phi^{\prime}=\phi-n \pi / 2$ in Step 4 , the coordinates of the strain are also rotated by $n \pi / 2$.

7. The coordinates for the macroscopic strain are rotated by $\theta$.

Steps 6 and 7 are expressed by

$\mathrm{C}^{H}=\mathbf{R}(\theta)^{T} \mathbf{R}(n \pi 2)^{T} \mathrm{C}_{\mathrm{arb}}^{H} \mathbf{R}(n \pi / 2) \mathbf{R}(\theta)$,

here

$$
\mathbf{R}(\theta)=\left[\begin{array}{ccc}
\cos ^{2} \theta & \sin ^{2} \theta & \cos \theta \sin \theta \\
\sin ^{2} \theta & \cos ^{2} \theta & -\cos \theta \sin \theta \\
-2 \cos \theta \sin \theta & 2 \cos \theta \sin \theta & \cos ^{2} \theta-\sin ^{2} \theta
\end{array}\right] .
$$

It should be noted that procedures 2 to 6 are not required if linear elastic problems are considered.

The ultimate strength of a 2-D cantilever with squaretype microstructures, as shown in Fig. 11, is calculated to verify the present interpolation technique. The cavity size parameters are specified as $a=0.2,0.4,0.6,0.8,1.0$, while the rotational angle as $\theta=0$. A prescribed displacement is given to the centre node on the right end of the lever, while the left side is fixed. The obtained load displacement curves are shown in Fig. 12. The results are plotted with the displacement as abscissa against the load as ordinate. The displacement $\delta$ is normalized by the length of the beam $L$. The load $P$ is normalized by $M_{p} / L$, where $M_{p}=\int_{-h / 2}^{h / 2} \sigma_{y} x t$ $\mathrm{d} x, t=1$. Dotted lines denote the results calculated directly by the homogenization method; solid lines indicate the solutions by the database. It can be observed that the results using the database are in good agreement with the solutions reached by the homogenization method. It should be noted that the result calculated by the homogenization method at $a=1.0$ (solid material) is exactly the same as that obtained by the normal elasto-plastic analysis.

The effectiveness of the database is shown by this numerical example. The computational times for the calculations using the database become $1 / 1000$ to $1 / 10000$ of those obtained directly by the homogenization method, depending on the degree of freedom of the unit cells shown in Fig. 5. Therefore, the database is indispensable to practical computation of nonlinear problems because not only the iterative calculations for the optimization but the load-incremental calculations are required.

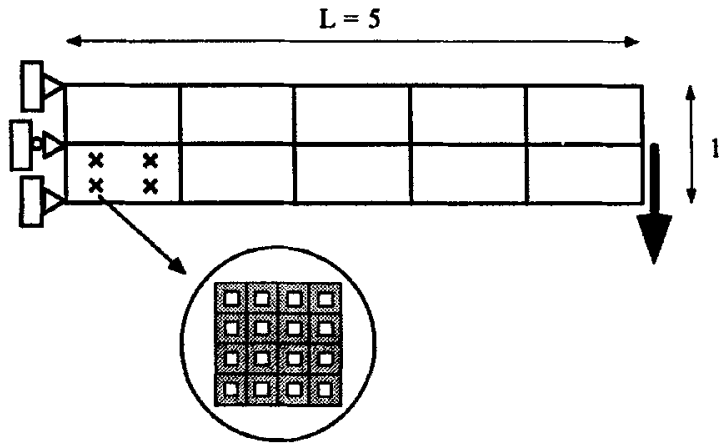

Fig. 11. 2-D cantilever subjected to a concentrated load

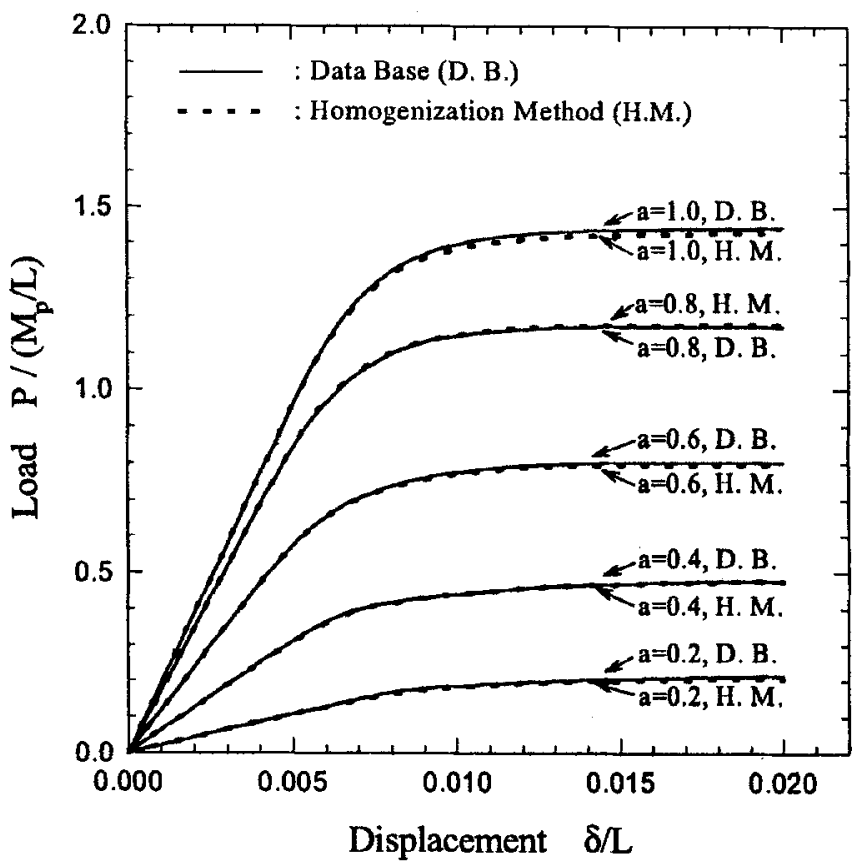

Fig. 12. Load displacement curves of a 2-D cantilever calculated by the homogenization method (dotted lines) and the data base (solid lines)

\section{Numerical examples for 2-D problems}

Numerical examples for nonlinear 2-D problems are shown in order to demonstrate the effectiveness of the present algorithm. Linear elastic optimized designs are also exhibited for comparison. Square-type microstructures are assumed in this section and the following sections. Material properties of the solid material in the microstructures are assumed as those of the mild steel. Prescribed displacements are given to the structures as applied loads in the following examples; therefore, the reaction forces are maximized by the optimization.

\subsection{Cantilever}

The optimization problem of a 2-D cantilever subjected to a nonlinear deformation is demonstrated. An admissible design domain $\Omega$ is assumed within the whole domain of the cantilever, as shown in Fig. 13. The admissible design domain is 
uniformly filled with square-type microstructures at the first iterative step of the optimization. A material restriction is $\Omega_{0} / \Omega=0.6$, where $\Omega_{0}$ is the total amount of solid material which can be distributed in the admissible domain.

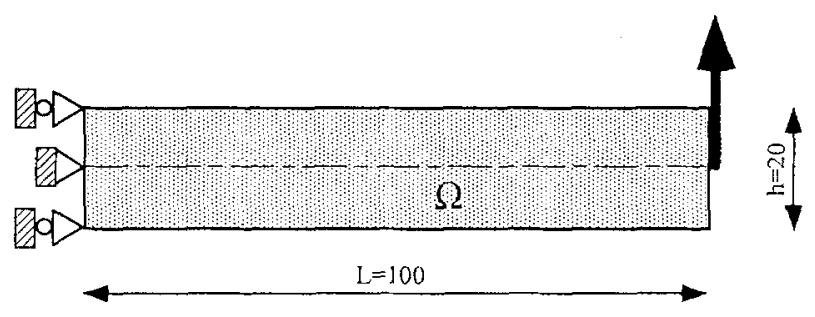

Fig. 13. Model of a cantilever subjected to a concentrated load with boundary conditions

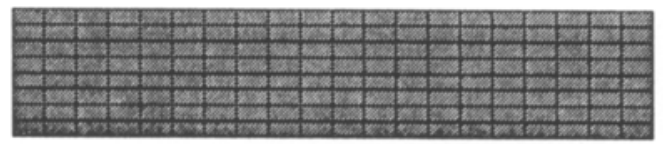

(a) $\mathrm{n}=1$

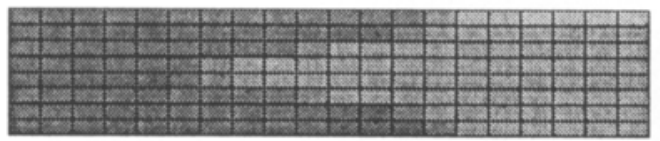

(b) $n=2$

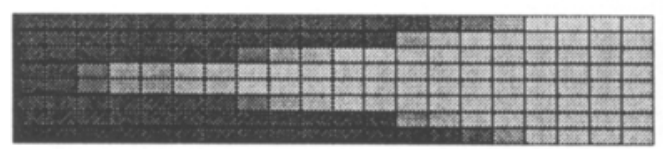

(c) $\mathrm{n}=5$

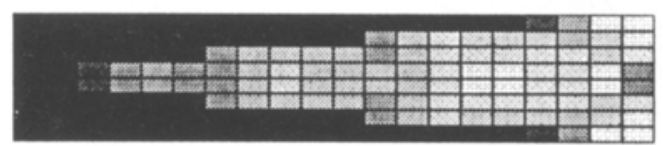

(d) $n=15$ (Optimized design)

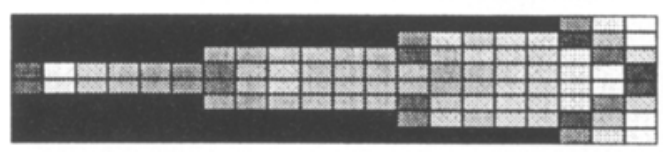

(e) Elastic optimization $(n=20)$

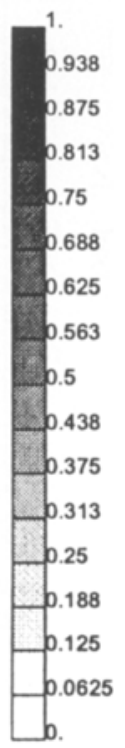

Fig. 14. History of the distribution of material densities of a cantilever. (a) $n=1$, (b) $n=2$, '(c) $n=4$, (d) $n=15$ (optimized design), (e) linear elastic optimization $(N=20)$

Figures 14a-d show the history of the material distribution at the same displacement for the nonlinear problem. The grey scale expresses the density of the solid material in each element, defined as $\int_{\Omega_{e}} 1-(1-a)^{2} \mathrm{~d} \Omega$. The number of the iteration of the optimization is expressed as " $n$ ". It can be seen macroscopically that material gradually moves to the area with higher strain density from the area with lower energy density during the iteration. Figure 14e shows the optimized design for the elastic problem. Both optimal designs become

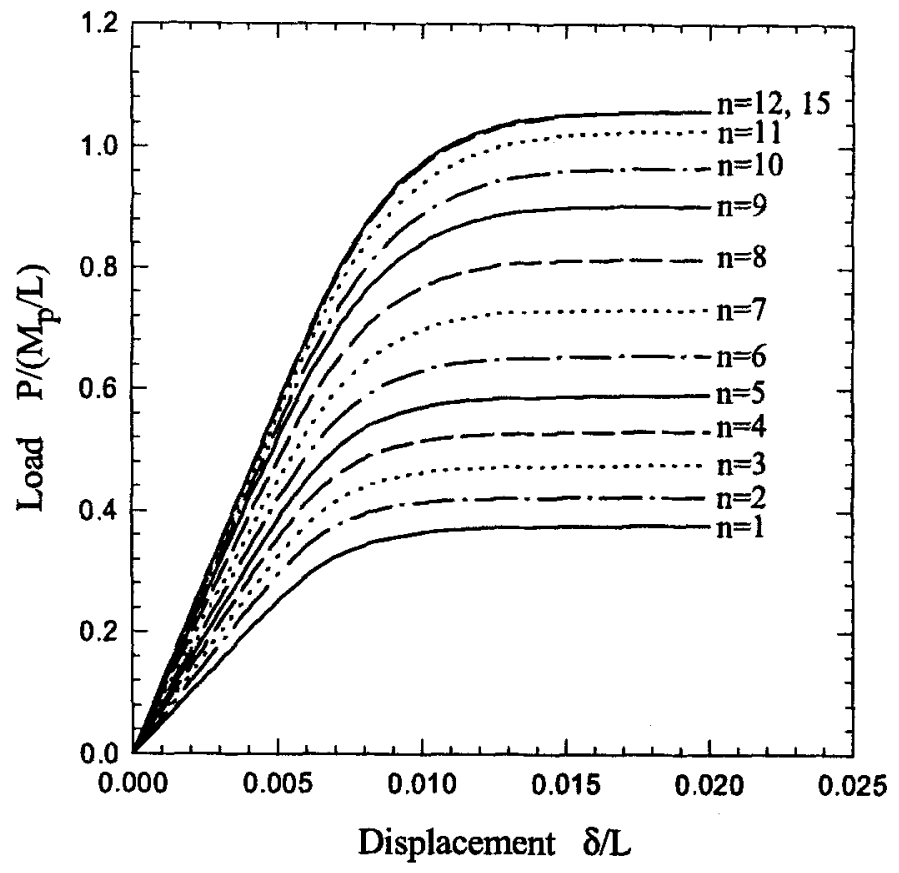

Fig. 15. History of load displacement curves of a cantilever

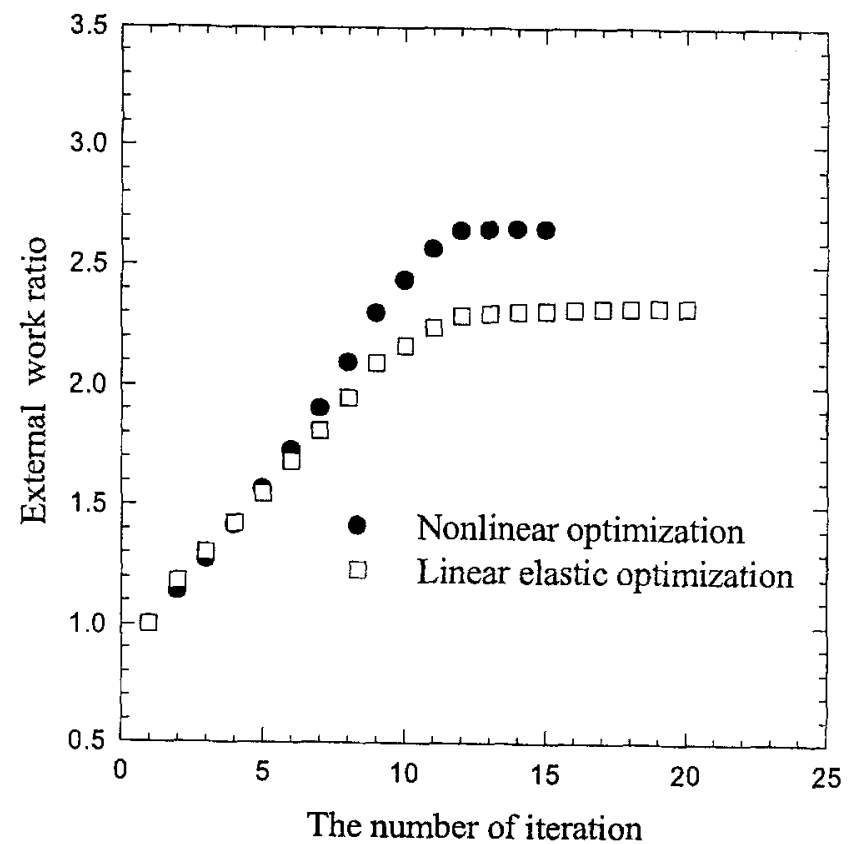

Fig. 16. History of external work of a cantilever

symmetrical with respect to the neutral axes. However, the greater amount of material is placed at the root of the lever for the nonlinear problem because higher material density at the root of the lever provides against the plastic hinge, in which most of the energy is absorbed. The difference of the optimal design for the nonlinear problem from that of the linear problem should become larger if the displacement is increased, because the optimal designs for nonlinear problems are dependent on the load while those for linear problems are not. It should be noted that we cannot cut off the low density area since the area sustains mainly shear stress. 
The history of the load displacement curves is shown in Fig. 15. The displacement is depicted in abscissa, and the reaction force is plotted in ordinate. The ultimate strength of the lever is gradually improved through the iteration since the prescribed displacement is applied. The ultimate strength of the optimized lever becomes 2.8 times as much as that of the initial design.

The history of the external work, or the objective function, is shown in Fig. 16. The horizontal axis expresses the number of iterations of the optimization. The vertical axis shows the applied external work from $\delta / L=0$ to $\delta / L=0.02$ at each iteration, which are normalized by the external work at the initial iteration. It can be seen that the function is maximized by the optimization since the prescribed load is applied, and converged smoothly for both problems.

\subsection{Extremely short lever}

The extremely short lever, as shown in Fig. 17, is optimized. The material restriction is $\Omega_{0} / \Omega=0.3$.

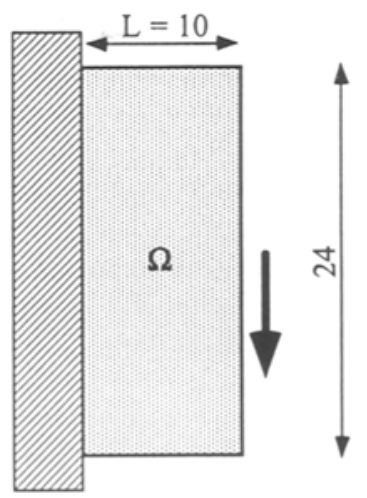

Fig. 17. Model of an extremely short lever subjected to concentrated load with boundary conditions

The optimal designs for the nonlinear problem and the linear elastic problem are depicted in Figs. 18a and b, respectively. It can be shown that the nonlinear design is the same as the linear elastic one, because the optimized designs become the structure whose members support only axial forces. The history of the load displacement curves are drastically changed by the optimization, shown in Fig. 19, because $\Omega_{0} / \Omega$ is rather low.

\section{Application to a thin shell}

The current optimization technique is applied to a thin shell based on the layered approach in this section. The optimized design of a cylindrical cantilever is shown as a numerical example.

\subsection{Thin shell element}

The bilinear shell element based on the layered approach is shown in Fig. 20. The global coordinates with primes are fixed in the space; elemental coordinates are rotated with the element. Components of the displacement of each layer

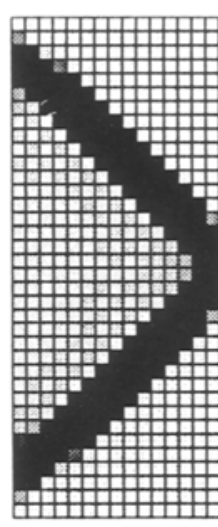

(a)

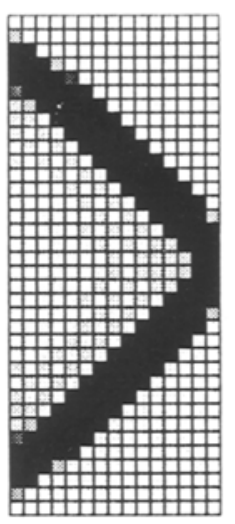

(b)
Fig. 18. Optimized designs of an extremely short lever. (a) Nonlinear optimization $(n=39)$, (b) linear elastic optimization $(n=38)$

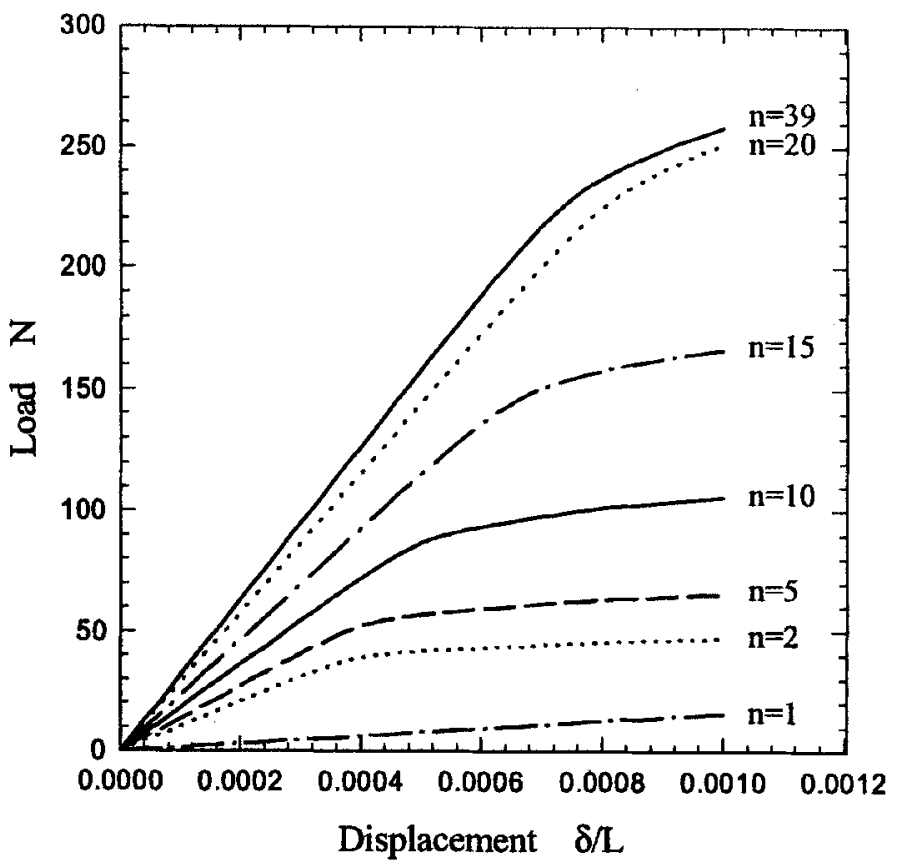

Fig. 19. History of load displacement curves of an extremely short lever

are expressed by

$\left\{\begin{array}{c}u_{1} \\ u_{2} \\ u_{3} \\ \theta_{1} \\ \theta_{2} \\ \theta_{3}\end{array}\right\}=\sum_{i=1}^{4} N^{(i)}(\xi, \eta)\left\{\begin{array}{c}u_{1}^{(i)} \\ u_{2}^{(i)} \\ u_{3}^{(i)} \\ \theta_{1}^{(i)} \\ \theta_{2}^{(i)} \\ \theta_{3}^{(i)}\end{array}\right\}+$ 
$\sum_{i=1}^{4} z N^{(i)}(\xi, \eta)\left\{\begin{array}{l}\theta_{2}^{(i)} \\ -\theta_{1}^{(i)} \\ 0 \\ 0 \\ 0 \\ 0\end{array}\right\}$

where $N^{(i)}(\xi, \eta)$ are shape functions, $i$ represents the nodal number in the element, and $z$ denotes the distance with sign between the neutral axis and the layer concerned.

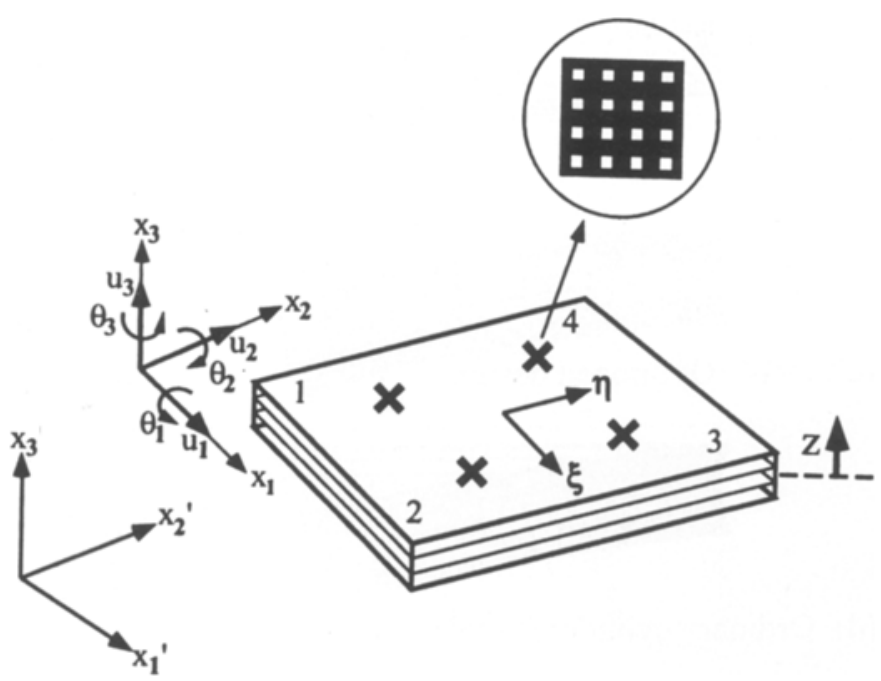

Fig. 20. Bilinear shell element based on the layered approach

The selective integration method is adopted, where the in-plane stress is evaluated at 2 by 2 Gaussian points on each layer, and the out-of-plane shear stress is calculated at the centre of the element to prevent the locking. Both constitutive equations for the in-plane stress and the out-of-plane shear stress are determined individually. If we assume 2-D periodic microstructures on each layer of the shell, the 2-D database can be applied to the in-plane stiffness on each layer by

$\sigma_{i j}=C_{i j k \ell}^{H} \varepsilon_{k \ell}, \quad(i, j, k, \ell=1,2)$,

where $C_{i j k \ell}^{H}$ is determined by interpolation of the 2-D database. If we suppose that the out-of-plane shear stress is always elastic, the constant stiffness is given as a penalty term, expressed by

$\sigma_{i j}=C^{P}\left(\varepsilon_{i j}+\varepsilon_{j i}\right) \quad(i=1,2 ; j=3)$.

Here $C^{P}$ is a proper constant, for example,

$C^{P}=\frac{E}{2(1+\nu)}$.

\subsection{Numerical example}

The optimized design of a cylindrical cantilever with four layers is demonstrated in this section. The whole domain of the cylinder is assumed as an admissible design domain, as depicted in Fig. 21. The left bottom of the cylinder is fixed; prescribed vertical displacements are uniformly applied at the all nodes on the right bottom. The material constraint is $\Omega_{0} / \Omega=0.6$.

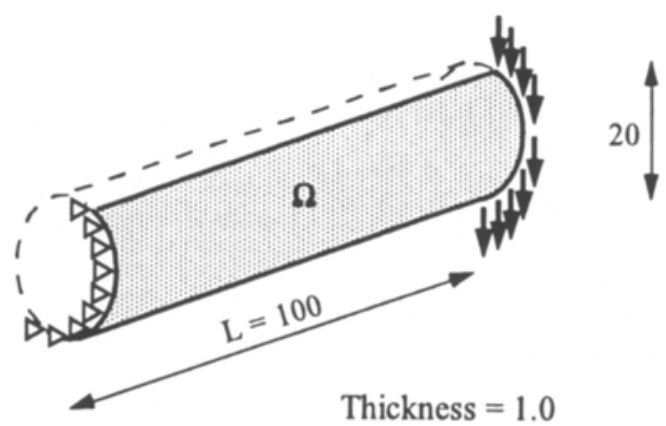

Fig. 21. Cylindrical cantilever subjected to distributed load

The optimized designs for the nonlinear problem and the linear elastic problem are shown in Figs. 22a and b. The displacements expressed in the figures are magnified by 10 and 750 for the nonlinear and the linear problems, respectively. It is observed that the material is placed mainly at the root and the outside regions from the neutral axis for both problems. The difference is the distribution near the neutral axis, which is the same result as that of the 2-D cantilever.

(a) Elasto-plastic problem
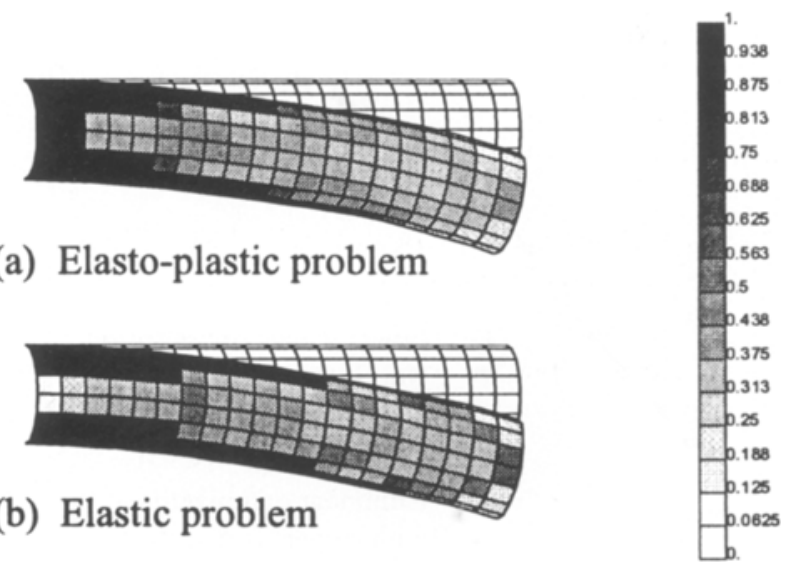

Fig. 22. Optimized designs of a cylindrical cantilever. (a) Nonlinear problem, (b) linear elastic problem

The histories of the load displacement curves are depicted in Fig. 23. It is observed that the load at the same displacement increases gradually during the iteration of the optimization, which shows similar results as those for 2-D problems.

The history of the objective function is shown in Fig. 24. The history of the nonlinear problem, denoted by black dots, has a slight vibration because of the nonlinearity. The history of the the linear elastic problem, depicted by white dots, converges very smoothly.

An effect of the out-of-plane shear stress $\sigma_{i j}(i=1,2$; $j=3$ ) is examined. The histories of the nonlinear and the linear elastic problems are shown by a black line and a dotted line in Fig. 24, respectively, where the out-of-plane stiffness 


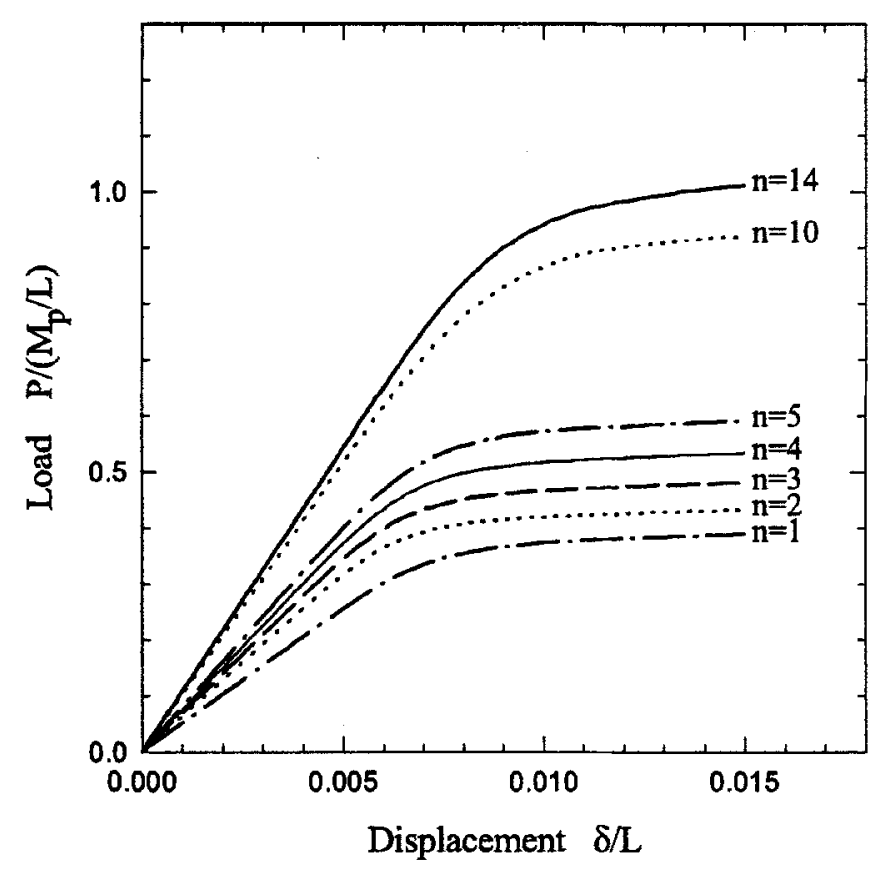

Fig. 23. History of the load displacement curves of a cylindrical cantilever

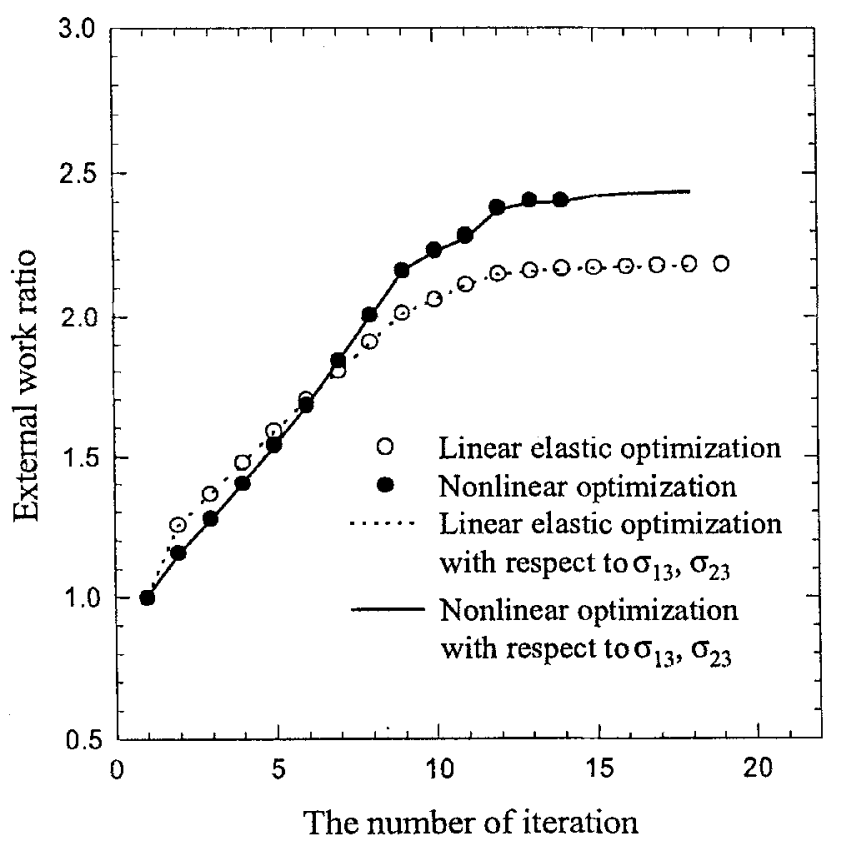

Fig. 24 History of the external work ratio of a cylindrical cantilever

is assumed to be proportional to the material density. The histories are almost the same as those of the original algorithm. In addition, the optimized designs also become the same as the original ones. It is shown that the influence of the out-of-plane shear stress is small and negligible.

The history of the distribution of the equivalent stress at the same displacement during the iteration for the optimization is shown in Figs. 25a-c. It can be seen that the stress increases gradually through the iteration, until the stress dis- tribution of the optimized cylinder becomes nearly the same as that of the ordinary cylinder which is made of solid material, as depicted in Fig. 25d. This shows that the optimized structure can sustain almost the same stress as that of an ordinary cylinder.

(a) $\mathrm{n}=1$

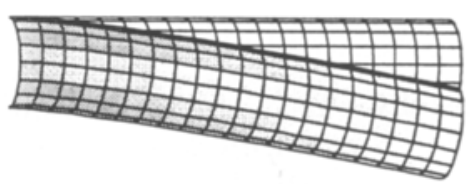

(b) $\mathrm{n}=5$
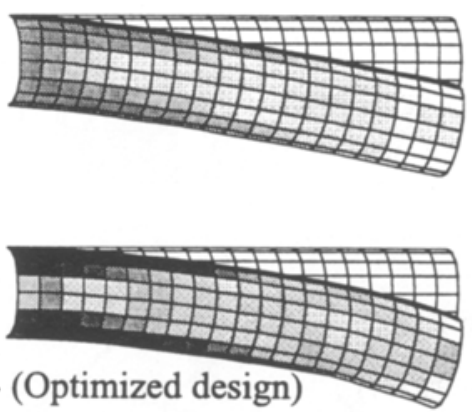

(c) $\mathrm{n}=14$ (Optimized design)

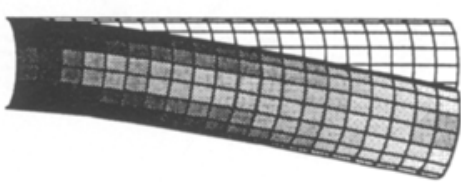

\section{(d) Ordinary cylinder (Solid material)}

Fig. 25. Distributions of equivalent stress of a cylindrical cantilever $(\delta / L=0.015)$. (a) $n=1$, (b) $n=5$, (c) $n=14$ (optimized design), and (d) ordinary cylinder (solid material)

The history of the yielded area is shown in Figs. 26a-d. The depth of the grey scale expresses the ratio of the number of the macroscopic plastic integration points to the number of the total integration points in the element. The definition of "macroscopic plastic" is one or more integration point(s) in the unit cell is/are yielded. At the first iteration for the optimization, the distribution is much different from that of the ordinary cylinder, shown in Fig. 26e, since square-type microstructures are uniformly distributed in the admissible domain. At the next iteration, the plastic area becomes drastically small because unit cells rotated to the directions of the principal stresses although the macroscopic material distribution is altered very slightly. Then, the plastic area becomes larger during the iteration. After the optimization, almost the whole admissible domain is yielded, as shown in Fig. 26d. The result indicates that the optimal material distribution is determined to sustain plastic deformation within the whole admissible domain.

\section{Application to finite deformation problems}

The present method is applied to finite deformation infinitesimal strain problems in this section. An optimized design of the reinforcement member is exhibited to demonstrate the effectiveness of our method. 


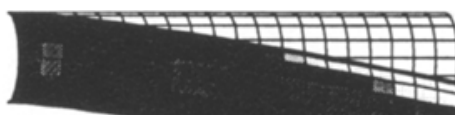

(a) $\mathrm{n}=1$

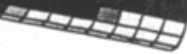

(b) $\mathrm{n}=2$

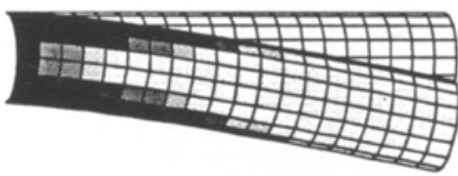

(c) $\mathrm{n}=5$
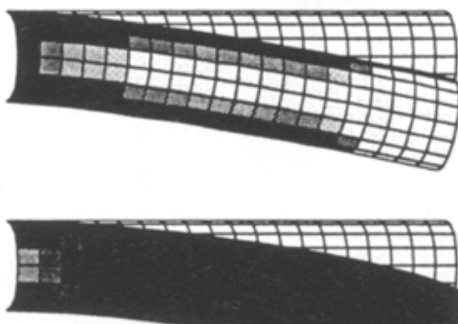

(d) $\mathrm{n}=14$ (Optimized design)

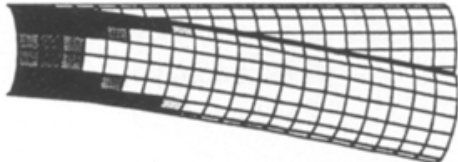

(e) Ordinary cylinder (Solid material)

Fig. 26. Plastic areas of a cylindrical cantilever $(\delta / L=0.015)$. (a) $n=1$, (b) $n=2$, (c) $n=5$, (d) $n=14$ (optimized design), and (e) ordinary cylinder (solid material)

\subsection{Finite deformation infinitesimal strain problems}

We assume finite deformation infinitesimal strain problems. According to the total Lagrangian approach, the macroscopic governing equation is defined by

$\int_{\Omega} \frac{\partial \delta u_{i}}{\partial X_{j}} \frac{\partial \dot{u}_{i}}{\partial X_{k}} T_{j k} \mathrm{~d} \Omega+$

$\int_{\Omega} \frac{\partial \delta u_{i}}{\partial X_{j}} \bar{C}_{i j k \ell}^{e p} \frac{\partial \dot{u}_{k}}{\partial X_{\ell}} \mathrm{d} \Omega=\int_{\Gamma_{t}} \delta u_{i} \dot{\tilde{t}}_{i} \mathrm{~d} \Gamma$,

where

$\bar{C}_{i j k \ell}^{e p}=C_{i j k \ell}^{e p}-\frac{1}{2}\left(\delta_{i \ell} T_{k j}+\delta_{i k} T_{\ell j}\right)-$

$\frac{1}{2}\left(T_{i k} \delta_{j \ell}+T_{i \ell} \delta_{j k}\right)$.

In (38), $\mathbf{u}$ denotes the displacement vector, $\mathbf{T}$ expresses the Cauchy stress tensor, $\tilde{\mathbf{t}}$ means the first Piola-Kirchhoff stress vector, $\delta$ exhibits a variational symbol, and $\Gamma_{t}$ denotes the mechanical boundary. In (39), $\delta$ represents Kronecker's delta.

The present database is applied to $\mathbf{C}^{e p}$ in (39) although the constitutive equation is, strictly speaking, dependent on the history of the stress, because it is well-known that the influence of the history upon the constitutive equation is not so large if the load is monotonously given. Practically, it has been shown that the difference is very small for the elasto-plastic problems between the solution calculated by the database and the exact solution calculated directly by the homogenization method with respect to the history of the stress, as depicted in Fig. 12. This fact is usually valid for finite deformation problems if some specified conditions, e.g. the buckling of thick members, etc., are excepted. In addition, since our algorithm distributes the material mainly at the area with higher strain energy density, almost the same design is obtained even if the stiffness is not very precisely calculated. Therefore, the current database is employed and the same interpolation technique is used for the finite deformation problems.

A plastic buckling problem of a thin shell with 6 layers is calculated to verify the matter, as shown in Fig. 27. The thickness is defined as $1 / 10$ of the width of an element. An initial imperfection is applied by a sinusoidal displacement whose amplitude is $1 / 1000$ of the length of the model. The obtained load displacement curves are depicted in Fig. 28. The ordinate represents the applied load; the abscissa denotes deflection. When solid material is assumed, $a=1.0$, it can be shown that the calculation with the database is almost the same as the solution calculated directly by the homogenization method, as depicted by white triangles and a dotted line, respectively. When $a=0.6$, the results are the same, as expressed by black triangles and a solid line. This example shows that the history of the stress, including local unloading, does not have a significant effect on the global stiffness of the thin shell subjected to a plastic deformation for finite deformation infinitesimal strain problems.

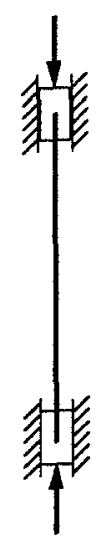

Thickness: 0.1

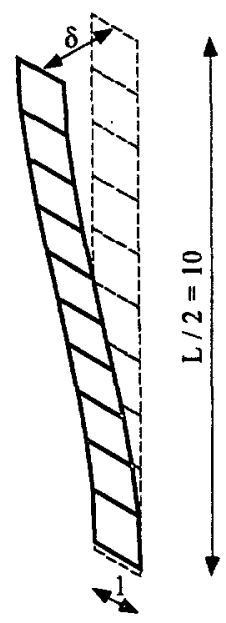

Fig. 27. Finite element model for buckling

\subsection{Numerical example}

A reinforcement member on the joining of a cylinder to an $I$-shaped beam is optimized. The model is divided into the admissible and the nonadmissible design domain, as depicted in Fig. 29. The hatched area consists of 8 layers, where the total thickness is $20 \mathrm{~mm}$; the central 4 layers are defined as the nonadmissible domain, where solid material is assumed; both upper 2 layers and lower 2 layers are defined as the admissible design domains in which the material constraint 


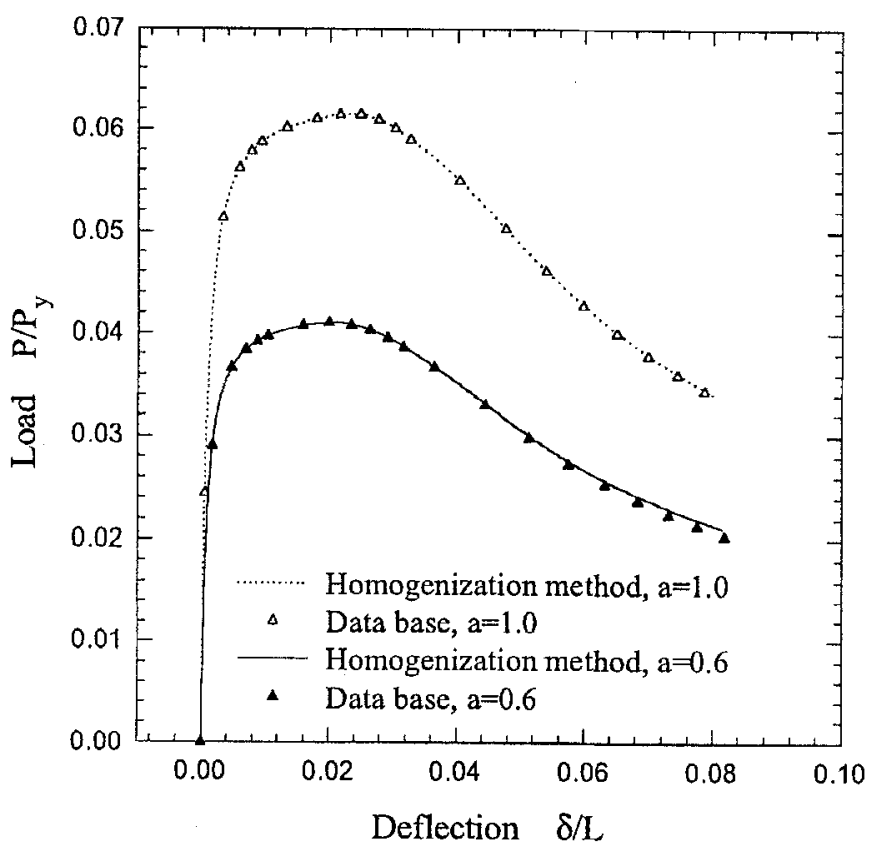

Fig. 28. Load displacement curves of buckling

is $\Omega_{0} / \Omega=0.3$. The nonhatched area is specified as the nonadmissible domain with 8 layers, where solid material is assumed; its total thickness is $10 \mathrm{~mm}$.

A dead load $P_{1}=P_{y} / 4$ is applied on the top of the cylinder, where $P_{y}=S \sigma_{y} ; S$ denotes the cross-section; $\sigma_{y}$ is the yield stress of the solid material. Then, the prescribed finite displacement is applied to the end of the beam by the load incremental method. The history of the load displacement curves is shown in Fig. 30. The longitudinal axis represents reaction force at the end of the beam; the transverse axis denotes the displacement on the top of the cylinder by the dead load. At the first iteration of the optimization $(n=1)$, the load decreases after the maximum point because partial buckling has occurred near the joining on the cylinder. During the following iterations, the buckling load becomes larger. At the final iteration, the load reduction almost disappears. In addition, it can be seen that the displacement is decreased by the optimization.

The optimized design of the reinforcement member is shown in Fig. 31a. It can be observed that almost all material is distributed near the joining on the cylinder in order to prevent the deflection which causes the buckling. It can also be seen that very little material is placed on the root of the flanges of the lever. This indicates that the strength of the original design of the lever has been sufficient. Additionally, the optimal design for the linear elastic infinitesimal problem is also exhibited in Fig. 31b. In this problem, the prescribed displacement on the lever is reduced to $1 / 1000$ of the previous problem. Note that the displacement which is depicted in the figure is multiplied by 100 . It is shown that material is distributed on the upper and the lower side of the joining on the cylinder, and the layout is much different from that for the finite deformation problems.

In the previous examples, some of the macroscopic outlines of the optimized structures were not so clear since mi-
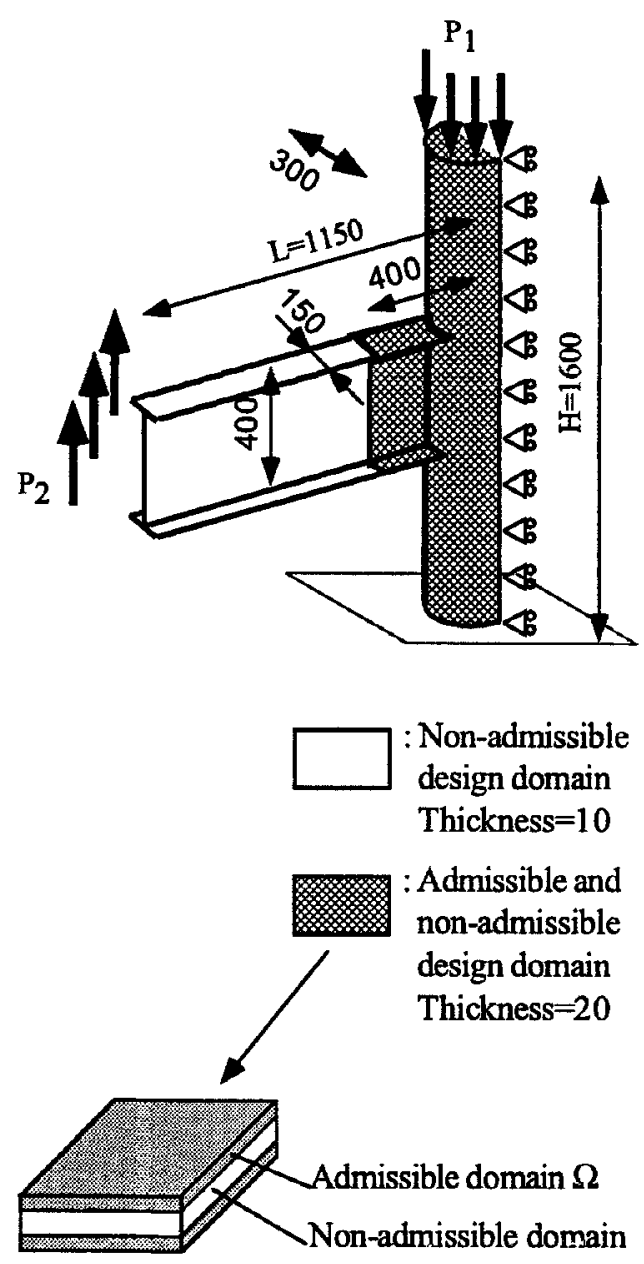

Fig. 29. Joining of a cylinder to an $I$-shaped beam

crostructures with larger cavities were distributed at the areas with lower strain energy density. In this problem, the obtained outlines are much clearer because the admissible domain is restricted to 4 out of 8 layers, and solid material is assumed at the remaining 4 layers.

\section{Concluding remarks}

A nonlinear optimization method for the shape and the topology problem was presented. The original algorithm is based on the generalized layout optimization method, where a macroscopic domain is supposed to consist of microstructures with periodic cavities; the sizes and the rotational angle of the cavities were defined as the design variables; the averaged material tensor of the macroscopic structure was computed by the homogenization method for the sensitivity analysis. We constructed the database of the material tensor and presented an interpolation technique to apply the optimization method to 2-D nonlinear problems. Our method was extended to a thin shell and its finite deformation problems. Several numerical examples showed:

1. The ultimate strength of the optimized structures became much higher than that of the initial designs; 


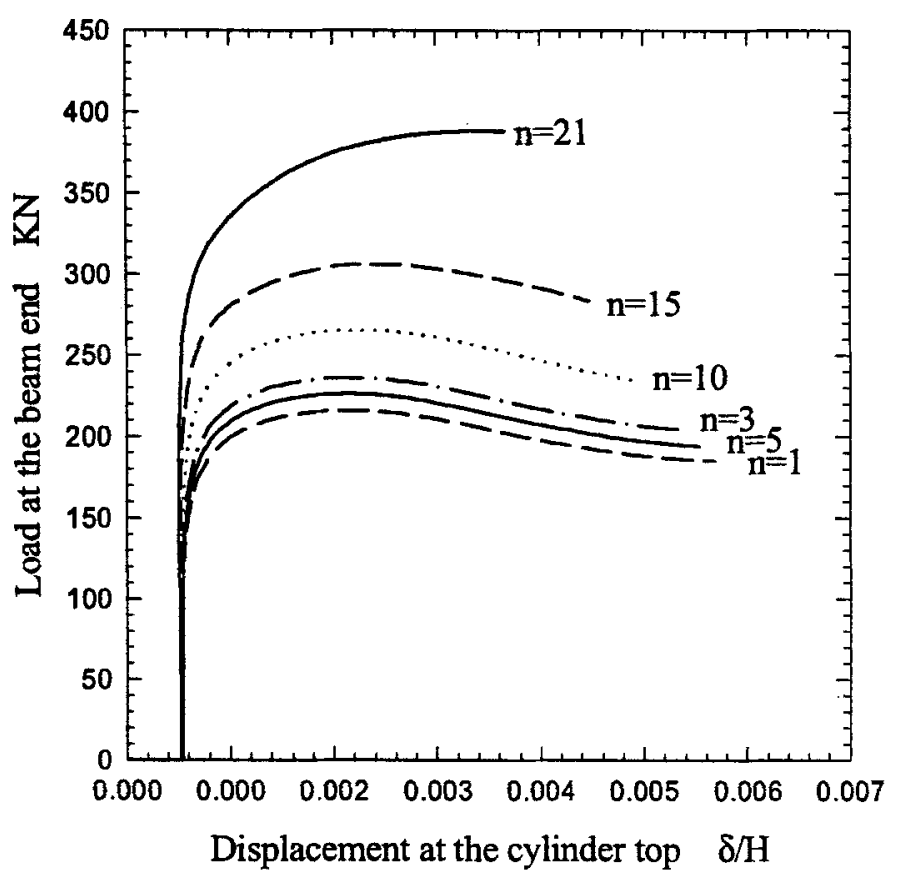

Fig. 30. History of the load displacement curves of the joining of a cylinder to an $I$-shaped beam

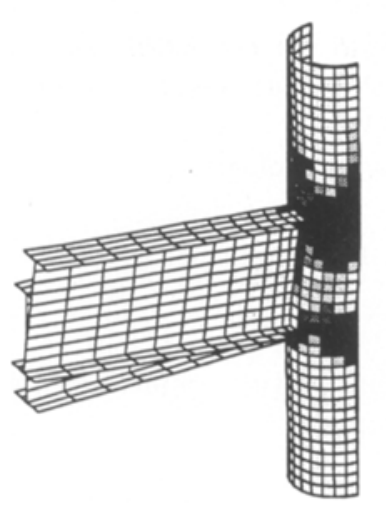

(a) Elasto-plastic finite deformation problem

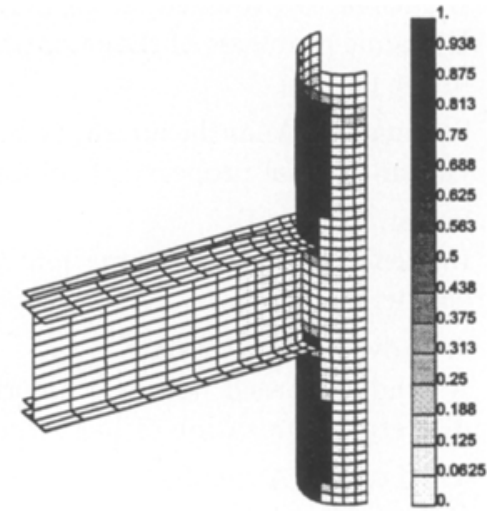

(b) Elastic infinitesimal problem
Fig. 31. Optimized designs of reinforcement members on the joining of a cylinder to an $I$-shaped beam. (a) Finite deformation problem, (b) linear elastic infinitesimal problem

2. The optimized designs for the linear elastic, the nonlinear, and the finite deformation problems were different from each other;

3. The optimal material distribution was determined to sustain plastic deformation within the whole admissible domain;

4. The influence of the out-of-plane shear stress of the thin shell upon the optimization was small and negligible;
5. When a reinforcement member was optimized, clearer macroscopic outlines were obtained.

\section{References}

Bendsøe, M.P.; Kikuchi, N. 1988: Generating optimal topologies in structural design using a homogenization method. Comp. Meth. Appl. Mech. Engrg. 71, 197-224

Bendsøe, M.P.; Diaz, A.R.; Kikuchi, N. 1993: Topology and generalized layout optimization of elastic structures. In: Bendsøe, M.P.; Mota Soares, C.A. (eds.) Topology design of structures, pp. 159-205. Dordrecht: Kluwer

Diaz, A.R.; Kikuchi, N. 1992: Solutions to shape and topology eigenvalue optimization problems using a homogenization method. Int. J. Num. Meth. Engrg. 35, 1487-1502

Guedes, J.M.; Kikuchi, N. 1990: Preprocessing and postprocessing for materials based on the homogenization method with adaptive finite element method. Comp. Meth. Appl. Mech. Engrg. 83, 143-198

Lions, J.L. 1981: Some method in the mathematical analysis of systems and their control. New York: Gordon and Breach Science Publ.

Ma, Z.-D.; Kikuchi. N.; Cheng, H.-C. 1995: Topological design for vibrating structures. Comp. Meth. Appl. Mech. Engrg. 121, 259-280

Ma, Z.-D.; Kikuchi, N.; Hagiwara, I. 1993: Structural topology and shape optimization for a frequency response problem. Comp. Mech. 13, 157-174

Suzuki, K.; Kikuchi, N. 1991: A homogenization method for shape and topology optimization. Comp. Meth. Appl. Mech. Engrg. 93, 291-318

Suzuki, K.; Kikuchi, N. 1992: Generalized layout optimization of three-dimensional shell structures. Geometric aspects of industrial design. Philadelphia: SIAM, 62-68

Terada, K.; Yuge, K.; Kikuchi, N. 1995: Elastoplastic analysis of composite materials using the homogenization method (1-st Report, Formulation, in Japanese). Trans. Japan Soc. Mech. Engineers, 61-590, A, 2199-2205

Terada, K.; Yuge, K.; Kikuchi, N. 1996: Elastoplastic analysis of composite materials using the homogenization method (2-nd Report, Numerical analysis, in Japanese). Trans. Japan Soc. Mech. Engineers, 62-601, A, 2072-2079

Yuge, K.; Kikuchi, N. 1995: Optimization of a frame structure subjected to a plastic deformation. Struct. Optim. 10, 197-208

Yuge, K.; Kikuchi, N. 1996: Optimal design of a two-dimensional structure subjected to a plastic deformation. Part 1, Formulation (in Japanese). Trans. Japan Soc. Mech. Engineers, 62-600, A, 1910-1917

Yuge, K.; Kikuchi, N.; Iwai, N. 1997: Optimal design of a twodimensional structure subjected to a plastic deformation (2-nd Report, Numerical Examples, in Japanese). Trans. Japan Soc. Mech. Engineers, 63-610, A, 1340-1347 